\title{
When two genes do not work properly
}

\author{
Corrado $\mathrm{G}^{*}$, Bruno $\mathrm{V}^{2}$ and Vizza $\mathrm{E}^{2}$ \\ ${ }^{1}$ Department of Woman, Child Health and Public Health, Gynecologic Oncology Unit, Fondazione Policlinico Universitario A. Gemelli, IRCCS, Rome, Italy \\ ${ }^{2}$ Department of Experimental Clinical Oncology, Gynecologic Oncology Unit, "Regina Elena" National Cancer Institute, IRCCS, Rome, Italy
}

BRCA1 and BRCA2 are human genes that produce tumour suppressor proteins. These proteins help repair damaged DNA and, therefore, play a role in ensuring the stability of the cell's genetic material. When either of these genes is mutated, or altered, such that its protein product is not made or does not function correctly, DNA damage may not be repaired properly. As a result, cells are more likely to develop additional genetic alterations that can lead to cancer. A harmful $B R C A 1$ or $B R C A 2$ mutation can be inherited from a person's mother or father. Each child of a parent who carries a mutation in one of these genes has a 50 percent chance of inheriting the mutation. The effects of mutations in BRCA1 and BRCA2 are seen even when a person's second copy of the gene is normal.

Specific inherited mutations in BRCA1 and BRCA2 increase the risk of female breast and ovarian cancers, and they have been associated with increased risks of several additional types of cancer. Together, BRCA1 and BRCA2 mutations account for about 20 to 25 percent of hereditary breast cancers [1] and about 5 to 10 percent of all breast cancers [2]. In addition, mutations in BRCA1 and BRCA2 account for around 15 percent of ovarian cancers overall [3]. Breast cancers associated with BRCA1 and BRCA2 mutations tend to develop at younger ages than sporadic breast cancers. Moreover, harmful mutations in $B R C A 1$ and $B R C A 2$ increase the risk of several cancers in addition to breast and ovarian cancer. BRCA1 mutations may increase a woman's risk of developing fallopian tube cancer and peritoneal cancer [4]. Men with BRCA2 mutations, and to a lesser extent $B R C A 1$ mutations, are also at increased risk of breast cancer [5]. Men with harmful BRCA1 or BRCA2 mutations have a higher risk of prostate cancer [6]. Men and women with $B R C A 1$ or BRCA2 mutations may be at increased risk of pancreatic cancer [7].

Several options are available for managing cancer risk in individuals who have a known harmful BRCA1 or BRCA 2 mutation. These include enhanced screening, prophylactic (risk-reducing) and surgery.

\section{Enhanced screening}

Some women who test positive for BRCA1 and BRCA2 mutations may choose to start screening at younger ages than the general population or have more frequent screening. For example, some experts recommend that women who carry a harmful $B R C A 1$ or BRCA2 mutation undergo clinical breast examinations beginning at age 25 to 35 years. And some expert groups recommend that women who carry such a mutation have a mammogram every year, beginning at age 25 to 35 years [8]. Unfortunately, no effective methods of ovarian cancer screening currently exist. Some groups recommend transvaginal ultrasound examinations, blood tests for the antigen CA125 , and clinical examinations for ovarian cancer screening in women with harmful BRCA1 or BRCA2 mutations, but none of these methods appears to detect ovarian tumours at an early enough stage to reduce the risk of dying from ovarian cancer [9]. The benefits of screening for breast and other cancers in men who carry harmful mutations in BRCA1 or $B R C A 2$ is also not known, but some expert groups recommend that men who are known to carry a harmful mutation undergo regular mammography as well as testing for prostate cancer.

Prophylactic (Risk-reducing) Surgery. Prophylactic surgery involves removing as much of the "at-risk" tissue as possible. Women may choose to have both breasts removed (bilateral prophylactic mastectomy) to reduce their risk of breast cancer. Surgery to remove a woman's ovaries and fallopian tubes (bilateral prophylactic salpingooophorectomy) can help reduce her risk of ovarian cancer. Removing the ovaries also reduces the risk of breast cancer in premenopausal women by eliminating a source of hormones that can fuel the growth of some types of breast cancer. Prophylactic surgery does not completely guarantee that cancer will not develop because not all at-risk tissue can be removed by these procedures. Some women have developed breast cancer, ovarian cancer, or primary peritoneal carcinomatosis (a type of cancer similar to ovarian cancer) even after prophylactic surgery. Nevertheless, the mortality reduction associated with this surgery is substantial: one study showed that women who underwent bilateral prophylactic salpingo-oophorectomy had a nearly 80 percent reduction in risk of dying from ovarian cancer and a more than 50 percent reduction in risk of dying from breast cancer [10]. No evidence is available regarding the effectiveness of bilateral prophylactic mastectomy in reducing breast cancer risk in men with a harmful $B R C A 1$ or BRCA2 mutation or a family history of breast cancer. Therefore, bilateral prophylactic mastectomy for men at high risk of breast cancer is considered an experimental procedure, and insurance companies will not normally cover it.

World Health Organization criteria for population screening for genetic predisposition to disease are that the disease is an important public health burden in the target population; that the risk of disease due to mutations in the screened genes is known; and that effective intervention exist to reduce morbidity and mortality among genetically susceptible individuals [11]. At present, the US Preventive Services Task Force (USPSTF) supports BRCA1 and BRCA2 testing based on family history and ancestry, but not for the entire female population, given the lack of data on risks for mutation carriers ascertained from the general population, rather than through a personal or family history of

${ }^{*}$ Correspondence to: Giacomo Corrado, Department of Woman, Child Health and Public Health, Gynecologic Oncology Unit, Fondazione Policlinico Universitario A. Gemelli, IRCCS, Rome, Italy, E-mail: giacomo.corrado@alice.it

Received: May 18, 2019; Accepted: June 04, 2019; Published: June 07, 2019 
cancer [12]. This position was correct based on the data then available. However, a just-completed study now provides evidence that supports offering BRCA1 and BRCA2 sequencing to all women. Populationbased screening enables mutation carriers to be identified independent of physician referral or family involvement. This is important, because at present, there is marked variability in practice in following the USPSTF guidelines. A recent survey revealed that only $19 \%$ of US primary care physicians accurately assessed family history for BRCA1/ BRCA2 testing [13].

Population-wide screening will require significant efforts to educate the public and to develop new counselling strategies, but this investment will both save women's lives and provide a model for other public health programs in genomic medicine. Women do not benefit by practices that "protect" them from information regarding their own health. They should have the choice to learn if they carry an actionable mutation in BRCA1 or BRCA2.

\section{References}

1. Easton DF (1999) How many more breast cancer predisposition genes are there? Breast Cancer Research 1: 14-17.

2. Campeau PM, Foulkes WD, Tischkowitz MD (208) Hereditary breast cancer: New genetic developments, new therapeutic avenues. Human Genetics 124: 31-42.

3. Pal T, Permuth-Wey J, Betts JA (2005) BRCA1 and BRCA2 mutations account for a large proportion of ovarian carcinoma cases. Cancer 104: 2807-2816.
4. Finch A, Beiner M, Lubinski J (2006) Salpingo-oophorectomy and the risk of ovarian, fallopian tube, and peritoneal cancers in women with a BRCA1 or BRCA2 mutation. JAMA 296: 185-192.

5. Tai YC, Domchek S, Parmigiani G, Chen S (2007) Breast cancer risk among male BRCA1 and BRCA2 mutation carriers. Journal of the National Cancer Institute 99: 1811-1814

6. Levy-Lahad E, Friedman E (2007) Cancer risks among BRCA1 and BRCA2 mutation carriers. British Journal of Cancer 96: 11-15.

7. Ferrone CR, Levine DA, Tang LH (2009) BRCA germline mutations in Jewish patients with pancreatic adenocarcinoma. Journal of Clinical Oncology 27: 433-438.

8. Burke W, Daly M, Garber J (1997) Recommendations for follow-up care of individuals with an inherited predisposition to cancer. II. BRCA1 and BRCA2. Cancer Genetics Studies Consortium. JAMA 277: 997-1003.

9. Evans DG, Gaarenstroom KN, Stirling D (2009) Screening for familial ovarian cancer: Poor survival of BRCA1/2 related cancers. Journal of Medical Genetics 46: 593-597.

10. Domchek SM, Friebel TM, Singer CF (2010) Association of risk-reducing surgery in BRCA1 or BRCA2 mutation carriers with cancer risk and mortality. JAMA 304: 967975

11. Khoury MJ, McCabe LL, McCabe ER (2003) Population screening in the age of genomic medicine. $N$ Engl J Med 348: 50-58

12. Moyer VA (2014) US Preventive Services Task Force. Risk assessment, genetic counseling, and genetic testing for BRCA-related cancer in women: U.S. Preventive Services Task Force recommendation statement. Ann Intern Med 160: 271-281.

13. Bellcross CA, Kolor K, Goddard KA, Coates RJ, Reyes M, Khoury MJ, et al. (2011) Awareness and utilization of BRCA1/2 testing among U.S. primary care physicians. Am J Presv Med 40: 61-66.

Copyright: (C2019 Corrado G. This is an open-access article distributed under the terms of the Creative Commons Attribution License, which permits unrestricted use, distribution, and reproduction in any medium, provided the original author and source are credited. 